L'optique s'impose comme

un outil de choix dans le

monde biomédical.

En particulier le laser, par ses

propriétés uniques, se révèle

irremplaçable pour la thérapie,

mais aussi pour l'imagerie.

Nous avons cherché ici

à illustrer deux notions qui

sont souvent évoquées

quand on parle de laser :

la cohérence spatiale

et la cohérence temporelle.

Nous montrons sur deux

exemples (la microscopie

confocale et la tomographie

de cohérence ou OCT),

qui ont leur place dans

les laboratoires et les hôpitaux, comment on peut réaliser des images qui sont de véritables coupes virtuelles, non invasives et non destructives,

dans des cellules ou des tissus du corps humain.

\title{
La cohérence de la lumière et l'imagerie des tissus du corps humain
}

A. Claude Boccara (claude.boccara@espci.fr)

Institut Langevin (ESPCI-ParisTech - CNRS - UP6 - UP7 - INSERM), ESPCI, 10 rue Vauquelin, 75231 Paris Cedex 05

La lumière peut traverser les tissus du corps humain, sauf dans le bleu et le vert où l'absorption du sang est importante, mais il n'est pas possible d'observer les structures autres que superficielles. Ainsi le médecin examine-t-il la peau à l'œil nu ou à la loupe, ou muni d'un endoscope pour profiter des ouvertures naturelles du corps humain. En effet, voir sous la surface se révèle délicat, car les tissus diffusent très fortement la lumière ; lorsque l'on souhaite réaliser une " coupe virtuelle ", c'est-à-dire non invasive, pour observer par exemple l'intérieur d'un grain de beauté sans prélèvement, il faut alors utiliser quelques-unes des possibilités offertes par les lasers dans le domaine de l'imagerie médicale.

Aujourd'hui, plusieurs approches permettent d'accéder aux structures cellulaires situées dans la profondeur des tissus et d'obtenir des images à l'échelle du microscope optique, c'est-à-dire avec une résolution de l'ordre du micron et un champ d'observation de l'ordre du millimètre.

Nous décrirons brièvement ici quelquesunes des méthodes les plus employées ces dernières années, qui ont largement contribué au développement de la biologie, mais encore peu à celui de la médecine. Nous ferons appel à deux propriétés des sources lumineuses et en particulier des lasers : la cohérence spatiale et la cohérence temporelle. Dans le premier cas, la " coupe » est obtenue par un effet géométrique; dans le second, nous décrirons une méthode qui tire profit des interférences dans un contexte très inhabituel : au lieu de travailler avec des composants optiques de qualité (comme les surfaces de miroirs), nous travaillerons avec les milieux « optiquement sales » que constituent les tissus du corps humain.

\section{Cohérences et découpage spatial}

Le domaine des ondes (et l'optique en particulier) fait appel à deux types de phénomènes pour définir la cohérence.

La cohérence spatiale est liée à la possibilité de focaliser un faisceau lumineux sur une toute petite surface dont la taille est voisine de la longueur d'onde : c'est la limite de diffraction. Si cette limite n'est pas atteinte avec les objectifs photographiques qui présentent trop d'aberrations, elle l'est couramment avec les objectifs de microscopes qui permettent de focaliser un faisceau laser visible sur un spot dont le rayon est inférieur au micromètre. Notons que si on voulait réaliser la même expérience avec un faisceau issu d'une autre source (incohérente spatialement), la puissance lumineuse que l'on pourrait focaliser sur le même spot serait mille à un million de fois moins élevée qu'avec un laser.

C'est cette très bonne focalisation qui est mise à profit dans la microscopie confocale : la figure 1 montre le principe de fonctionnement d'un tel microscope. Le laser est focalisé sur un diaphragme de petite taille, qui est lui-même focalisé par un objectif de microscope dans la profondeur d'un échantillon à examiner. La lumière de fluorescence ou de rétrodiffusion, qui est issue des couches qui ne se situent pas dans le plan de mise au point, est fortement atténuée, car elle ne se focalise pas sur le diaphragme de sortie qui est placé devant le détecteur (le plus souvent un photomultiplicateur). En balayant, à l'aide de miroirs (non représentés sur la figure), le plan de mise au point, on obtient l'image d'une " coupe " de l'échantillon. 


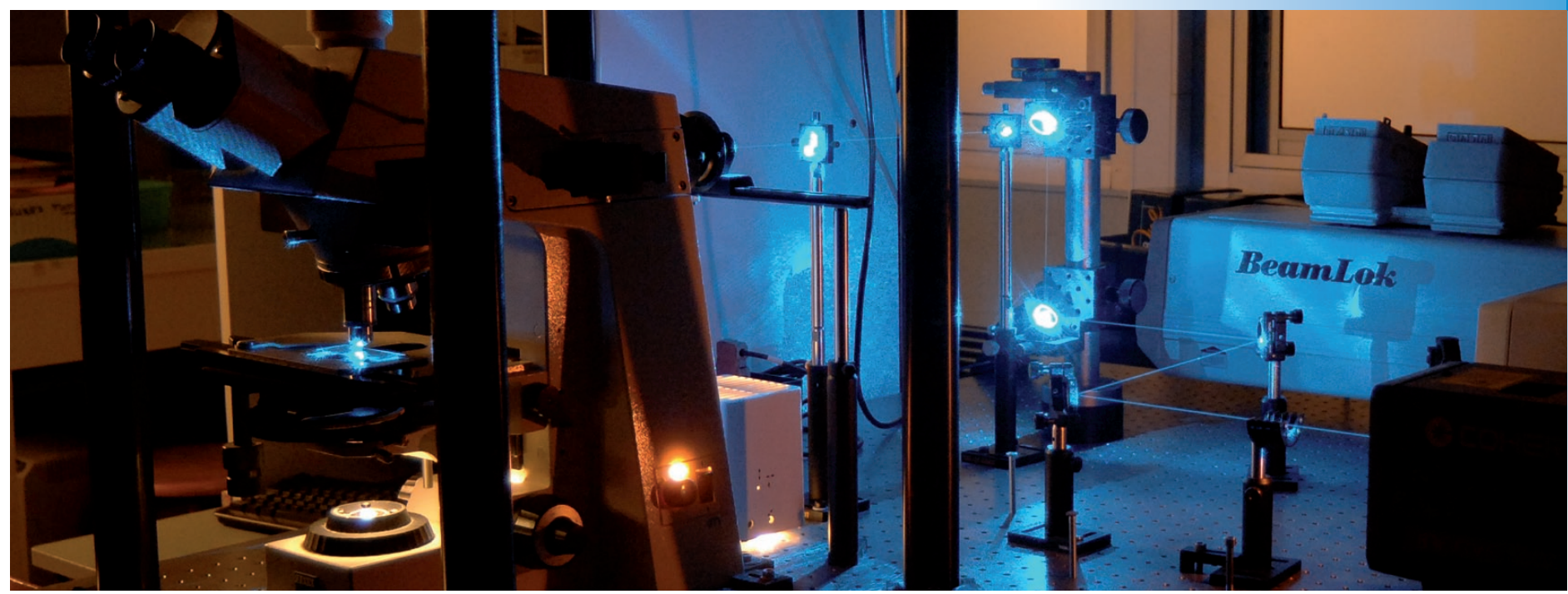

La lumière laser pour sonder le vivant : installation de microscopie optique en fluorescence (IPBS, Toulouse). @ cnRS Photothèque / VIALA Françoise.

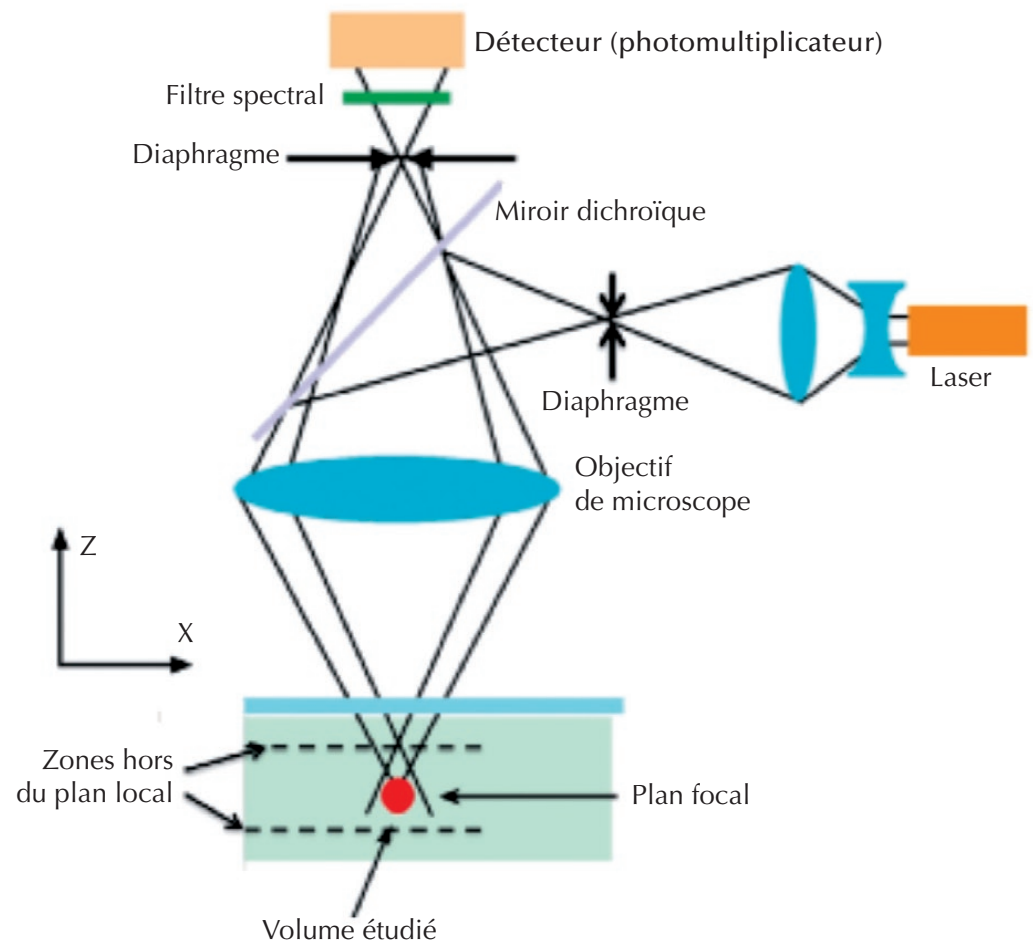

1. Principe de la microscopie confocale : les signaux de fluorescence ou de rétrodiffusion issus du plan focal de l'objectif passent par le diaphragme qui est devant le détecteur ; les signaux issus des autres plans sont bloqués par ce diaphragme.
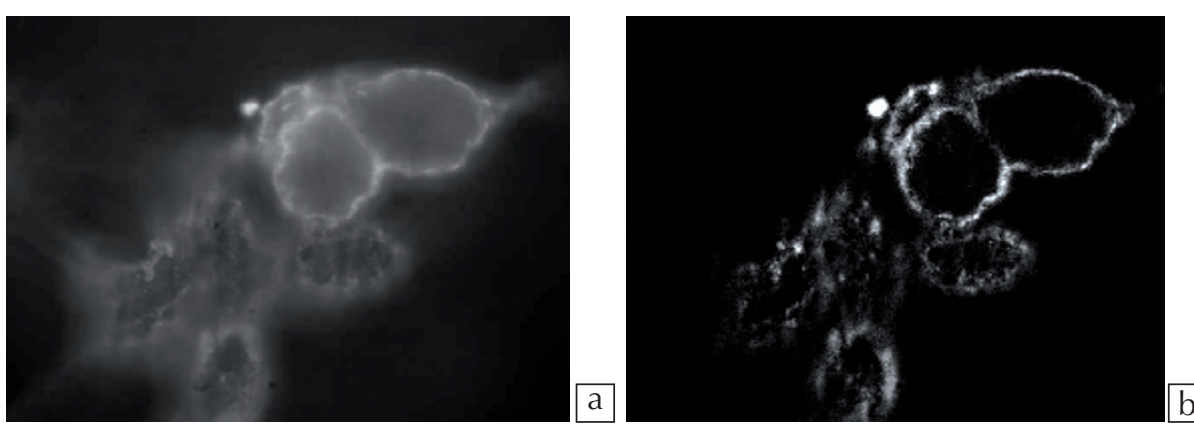

2. Images de cellules HEK (lignée de cellules de rein) dont la membrane est marquée par des nanocristaux fluorescents. (a) Image conventionnelle. (b) Coupe optique ; l'objectif est un 100x, de résolution transversale $260 \mathrm{~nm}$ et de résolution axiale (épaisseur de la « coupe ») $390 \mathrm{~nm}$. Le diamètre d'une cellule est de $13 \mu \mathrm{m}$.

La microscopie de fluorescence est un outil routinier en biologie : en effet, les marqueurs fluorescents (molécules de colorants, nanocristaux, protéines fluorescentes, etc.) peuvent cibler des cellules ou des structures spécifiques au sein des cellules elles-mêmes. Lorsqu'on observe une zone d'intérêt, on souhaite ne pas être gêné par les signaux qui sont issus des zones situées hors du plan de mise au point : c'est bien là l'objet de la microscopie confocale.

La figure 2 illustre cette possibilité de « sectionner » des cellules : on peut se rendre compte du flou qui vient des zones qui sont situées hors du plan de mise au point. Ici, c'est la membrane des cellules qui est marquée par des nanocristaux fluorescents. Notons cependant que si le développement de la microscopie confocale a été déterminant pour les progrès de la biologie, grâce au marquage sélectif par des fluorophores ou à l'utilisation d'animaux transgéniques, l'application au diagnostic médical est plus récente : parmi les succès de cette méthode, citons l'application à la dermatologie et l'utilisation d'endoscopes confocaux.

Cette possibilité de focaliser un faisceau laser sur une surface inférieure au micromètre carré est également mise à profit par les microscopies "non linéaires". Ces méthodes tirent en plus profit des impulsions très brèves, donc des puissances instantanées très grandes, que fournissent certains lasers dits "femtoseconde " (dont l'impulsion dure quelques millionièmes de milliardième de seconde) pour induire des transitions multiphotoniques ou engendrer des harmoniques de la fréquence de la lumière incidente qui ne se produisent qu'au foyer du microscope, là ou la densité de puissance est suffisante. 
La cohérence temporelle est liée à la monochromaticité de la source ou, ce qui est équivalent, à la possibilité d'obtenir des interférences avec de grandes différences de marche. La figure 3 représente le schéma de principe d'un interféromètre de Michelson : la lumière issue de la source est séparée en deux par un miroir partiellement réfléchissant ; elle se propage dans chaque bras, est réfléchie par des miroirs puis recombinée sur le miroir séparateur où les deux faisceaux interferent. Avec les lasers, la différence de chemin peut atteindre le million de kilomètres ; à l'opposé, avec les lasers à impulsions très brèves dont nous avons parlé plus haut, le domaine d'interférences observable sera de l'ordre de quelques micromètres.

C'est la faible cohérence temporelle qui est mise à profit dans la tomographie de cohérence (dite OCT pour Optical Coherence Tomography), dont le principe est représenté sur la figure 4.

La lumière rétrodiffusée par une tranche de l'échantillon ne pourra interférer avec le faisceau de l'autre bras de l'interféromètre que si la différence de marche est inférieure à la longueur de cohérence (la longueur sur laquelle la lumière se propage pendant la très brève impulsion ou le très faible temps de cohérence). Le balayage en profondeur est obtenu par déplacement du miroir de référence. Chaque "voxel» (élément de volume), le long d'une colonne, rétrodiffuse un signal qui interfere avec ce qui revient d'une position particulière du miroir de référence. Le détecteur mesure directement cet "écho ». On construit ainsi une colonne ; un balayage mécanique permet l'exploration transversale de l'échantillon et conduit aux coupes du type de celle qui est représentée sur la figure 5. Si l'épaisseur de coupe (résolution axiale) est liée à la longueur de cohérence de la source, la résolution transversale reste limitée par la diffraction.

C'est donc ainsi que sont réalisées des coupes de tissus. L'examen rétinien a été le premier succès de la méthode, aujourd'hui solidement établie en ophtalmologie (fig. 5).

Il n'est pas toujours nécessaire d'utiliser des lasers pour faire de l'OCT ; par exemple, des diodes superluminescentes (lasers à semi-conducteurs que l'on empêche d'osciller par un traitement antireflet des faces) sont couramment utilisées dans les

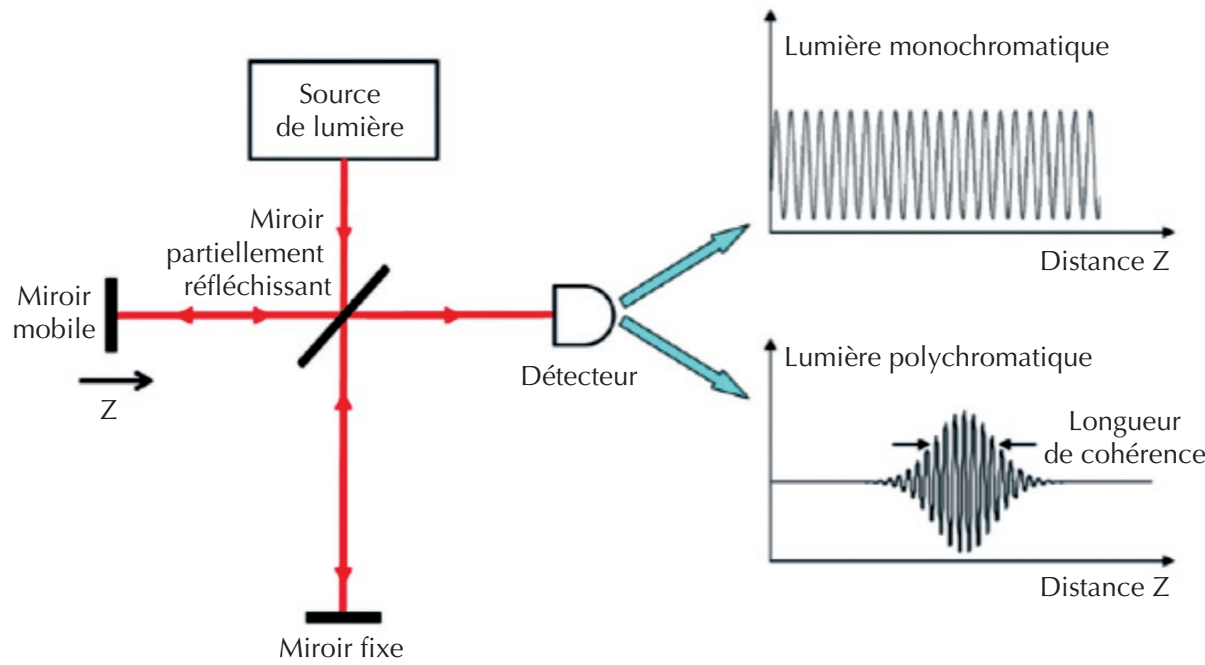

3. Interférences en lumière monochromatique et polychromatique observables avec un interféromètre de Michelson : dans le premier cas, on peut observer les franges avec des différences de marche très grandes (en haut à droite), alors que dans le second les franges s'amortissent rapidement (en bas à droite).

4. Montage de principe pour l'0CT le balayage axial est réalisé par déplacement du miroir de référence, le balayage transversal par déplacement du faisceau ou de l'échantillon.
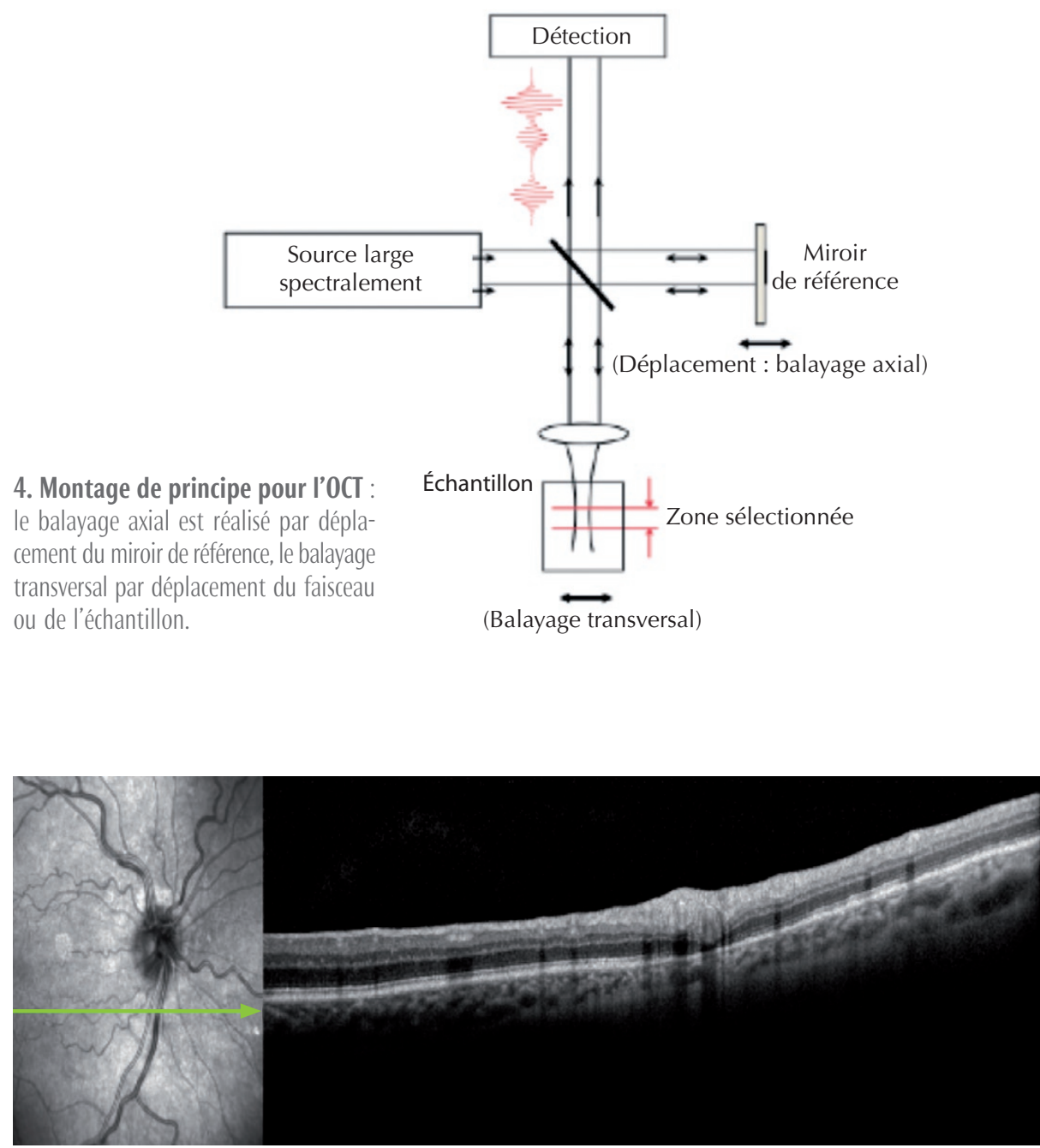

5. Examen de la rétine par 0CT. À gauche: image du fond de l'œil avec son réseau de vaisseaux sanguins. À droite: la coupe en profondeur prise par OCT le long de la flèche verte révèle les différentes couches de la rétine sur une épaisseur d'environ un demi-millimètre ; champ : $6 \times 3 \mathrm{~mm}^{2}$, résolution transversale : $14 \mu \mathrm{m}$, résolution axiale : 3,9 um. (Image Michel Pâques, Hôpital des Quinze-Vingts, Paris.) 


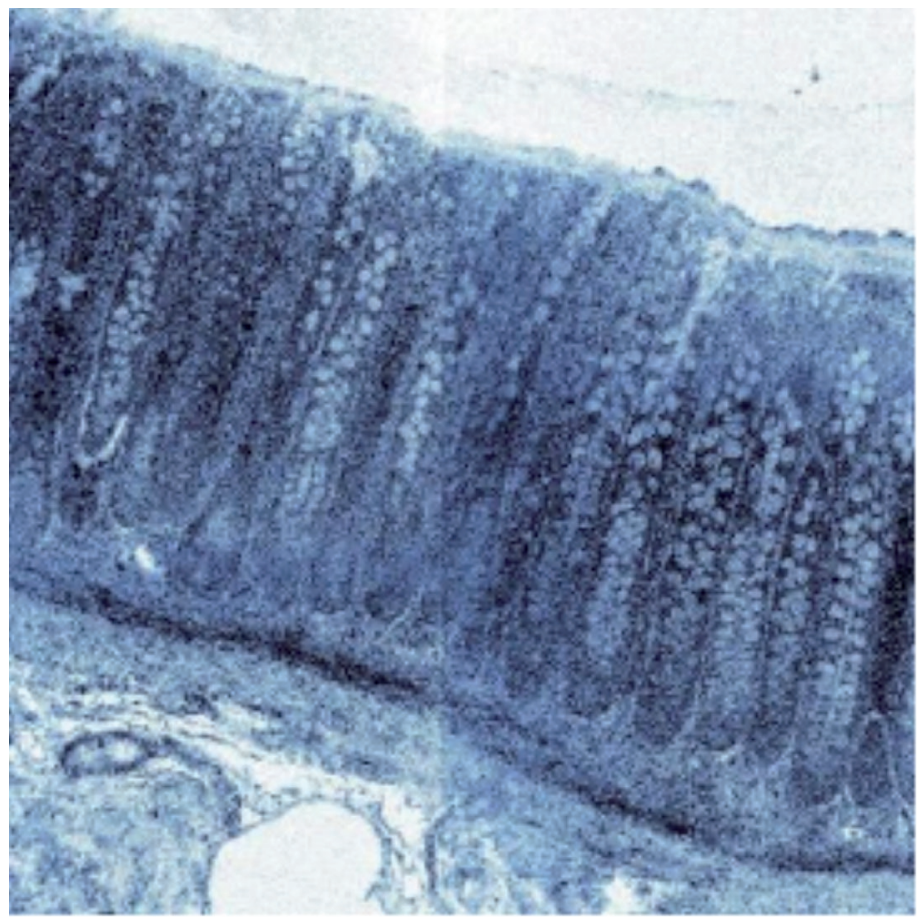

a

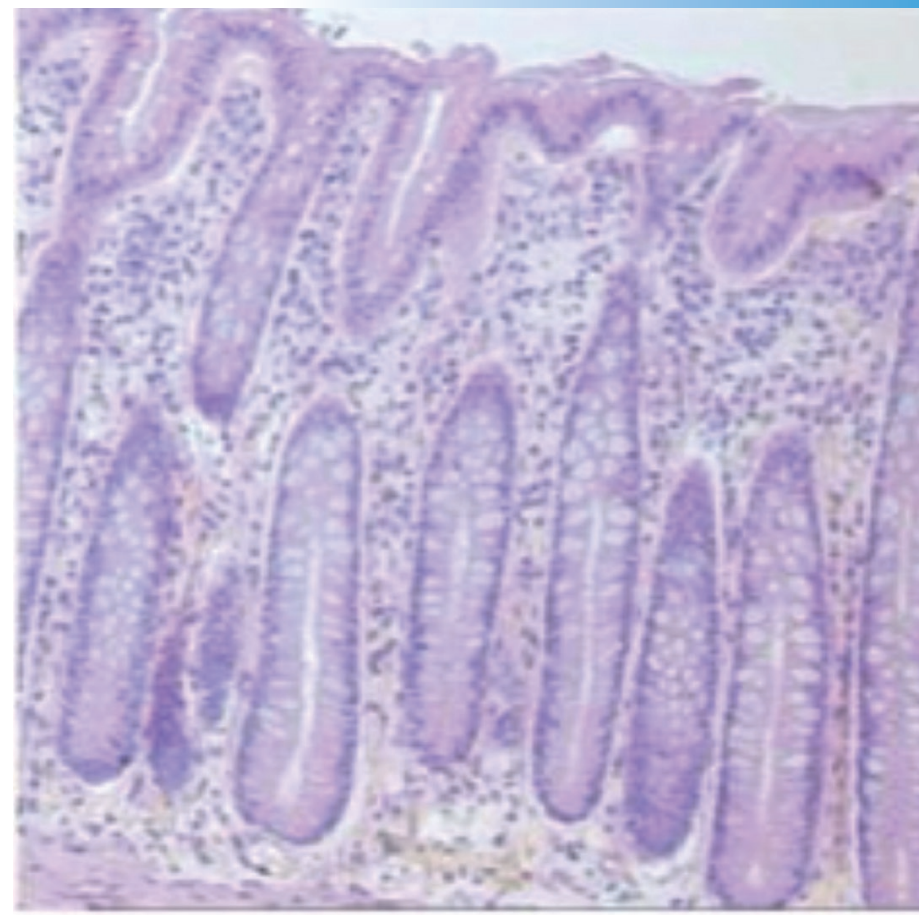

$\mathrm{b}$

6. Cellules épithéliales de la muqueuse du colon. (a) Image OCT, prise sur une pièce opératoire non coupée. (b) Coupe histologique, obtenue après une suite d'opérations qui peut prendre plusieurs jours ; le champ est de $1 \mathrm{~mm}^{2}$, la résolution transversale de 1,3 $\mu \mathrm{m}$ et la résolution axiale (coupe) de $1 \mu \mathrm{m}$. Images : www.lltech.fr.

montages commerciaux. Notons cependant que les images dont la qualité est la meilleure à ce jour ont été réalisées avec des lasers "ultra-brefs ", dont les impulsions durent de 6 à 12 femtosecondes (ce qui représente une coupe de l'ordre du micromètre d'épaisseur), non pas à cause de leur puissance, mais à cause de leur faible longueur de cohérence et leur faible bruit.

L'OCT permet de faire des mesures dimensionnelles (suivi du gonflement de la rétine), importantes pour le contrôle des sujets diabétiques, d'observer l'évolution de la dégénérescence maculaire, etc.

L'intérêt de cette approche, en plus de l'obtention de "sections", est de sélectionner les photons balistiques, qui sont les seuls à contribuer aux interférences, et d'éliminer les photons diffusés qui constituent un obstacle sévère pour l'observation des tissus biologiques.

En dehors du domaine privilégié de l'ophtalmologie, les deux autres domaines qui font l'objet d'études par OCT sont la dermatologie et le diagnostic préopératoire des tumeurs. Le but est ici soit de faire un examen que l'on peut qualifier de biopsie optique, c'est-à-dire, encore une fois, une coupe virtuelle non invasive et sans contact, soit d'examiner en un temps bref, pendant une opération chirurgicale, l'état d'une tumeur. Pour que ce type de diagnostic soit pertinent, il faut que les images fournies soient aussi voisines que possible des coupes histologiques que réalisent les anatomopathologistes. On peut se rendre compte sur la figure 6 de la pertinence des images obtenues par OCT.

\section{Conclusion et perspectives}

Nous avons cherché à illustrer ici quelques applications " pratiques ", à l'interface des mondes de la physique et du biomédical, de la notion " académique " de cohérence, qui s'est largement développée depuis l'apparition des lasers. Les cohérences dont nous avons parlé se retrouvent dans de très nombreux autres domaines : pièges optiques, génération de rayons $\mathrm{X}$ par lasers, détections hypersensibles (comme les ondes de gravitation), etc.

Il est clair aussi que les lasers médicaux jouent un rôle beaucoup plus large que celui que nous avons choisi de décrire.

En particulier, le laser "répare " le vivant (voir l'article de S. Mordon dans ce numéro) : citons les décollements de rétine, les cicatrisations de plaies, les résurfaçages de la cornée (correction de la myopie), le bistouri laser et l'épilation laser (qui représente une part notable du marché des lasers médicaux !).
Cette brève incursion dans le monde des lasers pour le médical nous montre que ce domaine, encore jeune, joue déjà un rôle qui ne cesse de prendre de l'importance. Les progrès attendus, tant en imagerie qu'en thérapie, sont liés à une maîtrise de la physique de ces milieux complexes que sont les tissus biologiques, de la technologie des sources et des détecteurs, et enfin à la réduction des barrières culturelles entre mondes scientifique, technique et médical. I

\section{Pour en savoir plus}

- Le Laser, coordonné par F. Bretenaker et N. Treps, EDP Sciences (2010).

- Optical Coherence Tomography: Technology and Applications, W. Drexler et J. G. Fujimoto, éditeurs, Springer (2008).

- J. C. Mertz, Introduction to Optical Microscopy, Roberts and Company Publisher (2009). 